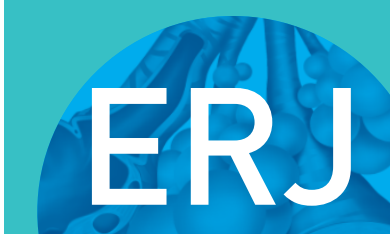

open research

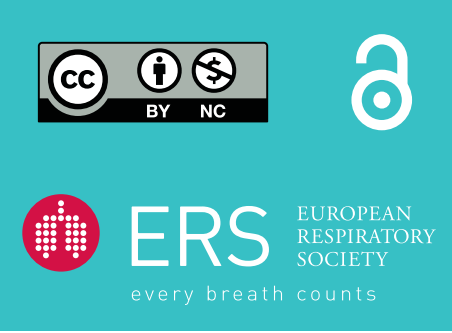

\section{Comprehensive evaluation of airway involvement in pulmonary sarcoidosis}

\section{To the Editor:}

Sarcoidosis most commonly affects the thoracic lymph nodes, lung parenchyma and airway in nearly two-thirds of patients [1]. We previously reported that airflow limitation in Japanese outpatients was associated with radiographic stage IV disease, older age, smoking and bronchovascular bundle thickening on high-resolution computed tomography (HRCT) [2]. Airway hyperresponsiveness (AHR) can be another potential predisposing factor of airflow limitation $[1,3]$. Impulse oscillometry (IOS) is an effort-independent and noninvasive method of assessing respiratory physiology and may detect more subtle changes than spirometry [4]. Thus, a multidisciplinary approach including AHR provocation test and IOS may provide novel insights into airway involvement of sarcoidosis compared to classical spirometric assessments. We conducted a prospective observational study to comprehensively evaluate airway involvement in patients with pulmonary sarcoidosis and investigate the impacts of various aspects of airway involvement on the long-term outcome. This study was approved by the Ethics Committee of Kyoto University (Kyoto, University; institutional board number E-530), with all subjects granting written informed consent.

We enrolled a total of 45 consecutive patients newly diagnosed with pulmonary sarcoidosis at Kyoto University Hospital between September 2008 and February 2012 for investigation. Grounds for exclusion were as follows: 1) systemic corticosteroid therapy within the preceding month; 2) history of adult asthma; 3) any other chronic lower airway disease, such as chronic obstructive pulmonary disease and bronchiectasis; 4) upper or lower airway infection within the preceding 4 weeks; 5) active neoplasm; or 6) connective tissue disease. The diagnosis of sarcoidosis was biopsy proven in $18(40 \%)$ patients and based on a combination of clinical and laboratory findings in the remaining 27 patients [5].

At the time of diagnosis, all subjects underwent chest radiography, measurement of serum angiotensinconverting enzyme, soluble interleukin-2 receptor and Krebs von den Lungen-6 and total immunoglobulin E, pulmonary function tests (PFTs), IOS (Masterscreen IOS-J; Jaeger, Wurzburg, Germany) [4], exhaled nitric oxide fraction $(\mathrm{FeNO})$ measurement at expiratory flow rates of $50 \mathrm{~mL} \cdot \mathrm{s}^{-1}$ (NOA 280; Sievers, Boulder, CO, USA) [6] and AHR provocation test [7, 8]. HRCT of the chest (Aquilion 64; Toshiba, Tokyo, Japan) and bronchoalveolar lavage (BAL) were also performed in $44(98 \%)$ and $36(80 \%)$ patients, respectively [5]. The extent of organ involvement was assessed by the ACCESS (A Case-Control Epidemiologic Study of Sarcoidosis) Organ Involvement Index [9]. The AHR test was performed according to the single concentration four-step dosimeter protocol for methacholine [7,8]. A single concentration of methacholine-chloride $\left(16 \mathrm{mg} \cdot \mathrm{mL}^{-1}, 1.6 \%\right)$ was administered through a nebuliser (MedicAid Pro Nebulizer; Jaeger) at the output of $240 \mu \mathrm{L} \cdot \mathrm{min}^{-1}$. In IOS, rectangular mechanical impulses in the frequency range of $5-35 \mathrm{~Hz}$ were applied to the respiratory system through a mouthpiece during spontaneous breathing. Resultant pressure and flow signals were analysed to determine respiratory resistance values at $5 \mathrm{~Hz}$ and $20 \mathrm{~Hz}$ (R5 and R20, respectively), reactance at $5 \mathrm{~Hz}(\mathrm{X} 5)$ and area of reactance $(\mathrm{AX})[4,10]$. HRCT images $(0.5-\mathrm{mm}$ slices) were analysed to determine airway wall area of the right apical bronchus and tracheal luminal area at the upper margin of aortic arch, using a modified in-house software as previously described [11].

Of the 45 patients enrolled, AHR was detected in eight (18\%) out of 45 patients. Clinical manifestations, serum biomarkers, IOS, PFT, FeNO, HRCT indices and BAL findings were compared between patients with

$@$ ERSpublications

IOS can predict airway hyperresponsiveness and long-term outcome in patients with pulmonary sarcoidosis http://ow.ly/bkQH307VD4m

Cite this article as: Tanizawa $\mathrm{K}$, Handa $\mathrm{T}$, Nagai S, et al. Comprehensive evaluation of airway involvement in pulmonary sarcoidosis. ERJ Open Res 2017; 3: 00105-2016 [https://doi.org/10.1183/ 23120541.00105-2016].

Copyright $\odot$ ERS 2017. This article is open access and distributed under the terms of the Creative Commons Attribution Non-Commercial Licence 4.0. 
and without AHR (table 1). Respiratory symptoms (cough, sputum production, wheezing or dyspnoea on exertion) were more prevalent in AHR-positive patients. Among IOS indices, R5 and R20 were significantly higher in AHR-positive patients, whereas R5-R20 and X5 values were similar, regardless of

TABLE 1 Comparison between patients with and without airway hyperreactivity (AHR)

\begin{tabular}{|c|c|c|c|}
\hline AHR & Positive & Negative & p-value \\
\hline Subjects & $n=8$ & $n=37$ & \\
\hline Age years & $39.5(26.8-70.3)$ & $51(35.5-60.0)$ & NS \\
\hline Males & 3 (38) & $16(43)$ & NS \\
\hline Height $\mathrm{cm}$ & $157(149-174)$ & $162(157-170)$ & NS \\
\hline Weight kg & $52.9(45.3-78.5)$ & $58.0(51.3-63.0)$ & NS \\
\hline Current or past smoker & 3 (38) & $20(54)$ & NS \\
\hline Smoking history pack-years & $0(0-10)$ & $1.5(0-37.4)$ & \\
\hline Histologic confirmation & $4(50)$ & $14(38)$ & NS \\
\hline Extrapulmonary involvement & $5(65)$ & $24(63)$ & NS \\
\hline Radiographic stage I/II/III/IV & $2(25) / 6(75) / 0(0) / 0(0)$ & $11(30) / 23(62) / 3(8) / 0(0)$ & NS \\
\hline Respiratory symptoms & $6(75)$ & $10(27)$ & 0.02 \\
\hline $\mathrm{mMRC}^{\#} 1 / 2 / 3 / 4 / 5$ & $5(63) / 2(25) / 1(13) / 0(0) / 0(0)$ & $31(84) / 5(14) / 1(3) / 0(0) / 0(0)$ & NS \\
\hline \multicolumn{4}{|l|}{ Serum biomarkers } \\
\hline ACE IU. $\mathrm{L}^{-1}$ & 18.9 (12.5-25.9) & 16.7 (14.0-26.3) & NS \\
\hline $\mathrm{sIL}-2 \mathrm{R} \mathrm{U} \cdot \mathrm{mL}^{-1}$ & $904(710-1410)$ & $532(409-889)$ & NS \\
\hline $\mathrm{KL}-6 \mathrm{U} \cdot \mathrm{mL}^{-1}$ & $341(251-451)$ & $271(214-361)$ & NS \\
\hline $\lg E \mathrm{IU} \cdot \mathrm{mL}^{-1}$ & $110(100-370)$ & $92(26-338)$ & NS \\
\hline \multicolumn{4}{|l|}{ Impulse oscillometry } \\
\hline $\mathrm{R} 5 \mathrm{kPa} \cdot \mathrm{L}^{-1} \cdot \mathrm{s}^{-1}$ & $0.33(0.28-0.43)$ & $0.23(0.19-0.31)$ & 0.01 \\
\hline $\mathrm{R} 20 \mathrm{kPa} \cdot \mathrm{L}^{-1} \cdot \mathrm{s}^{-1}$ & $0.29(0.24-0.35)$ & $0.21(0.17-0.28)$ & 0.02 \\
\hline $\mathrm{R} 5-\mathrm{R} 20 \mathrm{kPa} \cdot \mathrm{L}^{-1} \cdot \mathrm{s}^{-1}$ & $0.05(0.01-0.12)$ & $0.03(0.0-0.04)$ & NS \\
\hline $\mathrm{X} 5 \mathrm{kPa} \cdot \mathrm{L}^{-1} \cdot \mathrm{s}^{-1}$ & $-0.12(-0.20--0.08)$ & $-0.09(-0.13--0.07)$ & NS \\
\hline $\mathrm{AX} \mathrm{kPa} \cdot \mathrm{L}^{-1}$ & $0.27(0.21-1.15)$ & $0.17(0.13-0.38)$ & NS \\
\hline \multicolumn{4}{|l|}{ Pulmonary function } \\
\hline FVC \% pred & $102.6(91.5-107.2)$ & $111.0(99.9-122.4)$ & NS \\
\hline FEV $1 \%$ pred & 94.4 (91.2-98.3) & $106.9(92.0-116.1)$ & NS \\
\hline $\mathrm{FEV}_{1} / \mathrm{FVC} \%$ & $75.8(71.0-86.1)$ & $81.3(75.4-90.2)$ & NS \\
\hline $\mathrm{FEV}_{1} / \mathrm{FVC}<70 \%$ & $1(13)$ & $2(5)$ & NS \\
\hline PEF \% pred & $80.4(58.6-94.4)$ & $101.6(86.2-113.8)$ & 0.01 \\
\hline MMF \% pred & 67.7 (45.5-91.8) & 81.0 (56.5-104.9) & NS \\
\hline FRC \% pred & 105.0 (97.5-116.8) & $114.4(95.1-121.4)$ & NS \\
\hline RV \% pred & 117.3 (103.7-139.8) & 101.8 (83.3-123.8) & NS \\
\hline TLC $\%$ pred & 101.2 (89.9-123.4) & $101.9(87.5-115.6)$ & NS \\
\hline RV/TLC \% & $29.8(24.9-35.9)$ & 30.9 (25.3-33.8) & NS \\
\hline DLco \% pred & $77.4(64.4-86.3)$ & $78.1(68.6-89.6)$ & NS \\
\hline eNO & $29.4(18.2-47.7)$ & $21.5(16.8-124.0)$ & NS \\
\hline \multicolumn{4}{|l|}{ HRCT analyses } \\
\hline $\mathrm{Ai} \mathrm{mm^{2 }}$ & $22.3(15.7-33.2), n=7$ & $23.7(18.0-34.4), n=36$ & NS \\
\hline $\mathrm{Ai} / \mathrm{BSA} \times 10^{-6}$ & $13.6(10.9-18.5), n=7$ & $14.2(11.6-19.9), n=36$ & NS \\
\hline WA $\%$ & $60.5(55.3-66.5), n=5$ & $56.5(52.7-60.8), n=33$ & NS \\
\hline Atr $\mathrm{mm}^{2}$ & $225.2(176.5-257.5), n=7$ & $249.1(214.0-319.4), n=37$ & NS \\
\hline Atr $/$ BSA $\times 10^{-6}$ & $114.3(98.6-168.2), n=7$ & $156.1(133.6-189.9), n=37$ & NS \\
\hline BAL & $n=6$ & $\mathrm{n}=30$ & \\
\hline Recovered cells $\times 10^{5} \mathrm{~mL}^{-1}$ & $1.80(1.35-2.40)$ & $1.90(1.48-2.03)$ & NS \\
\hline Lymphocytes \% & $39.9(25.2-59.6)$ & $25.4(13.9-44.2)$ & NS \\
\hline Eosinophils \% & $0.3(0.0-1.1)$ & $0.0(0.0-0.0)$ & NS \\
\hline CD4/CD8 ratio & $2.90(2.71-11.2)$ & $5.90(4.33-7.53)$ & NS \\
\hline
\end{tabular}

Data are presented as median (interquartile range) or $\mathrm{n}(\%)$, unless otherwise stated. mMRC: modified Medical Research Council dyspnoea scale; ACE: angiotensin-converting enzyme; sIL-2R: soluble interleukin-2 receptor; KL-6: Krebs von den Lungen-6; IgE: immunoglobulin $\mathrm{E}$; R5: resistance at $5 \mathrm{~Hz}$; R20: resistance at $20 \mathrm{~Hz}$; X5: reactance at $5 \mathrm{~Hz}$; XA: area of reactance; FVC: forced vital capacity; FEV1: forced expiratory volume in $1 \mathrm{~s}$; PEF: peak expiratory flow rate; MMF: maximum mid-expiratory flow rate; FRC: functional residual capacity; RV: residual volume; TLC: total lung capacity; DLCO: diffusing capacity of the lung for carbon monoxide; eNO: exhaled nitric oxide; HRCT: high-resolution computed tomography; Ai: luminal area of right apical bronchus; BSA: body surface area; WA: wall area of right apical bronchus; Atr: tracheal luminal area; BAL: bronchoalveolar lavage; NS: not significant. ${ }^{\#}$ : theoretical range 1-5. 
AHR status. Likewise, PFTs and FeNO levels did not differ significantly between the two groups, except for the percentage of predicted peak expiratory flow rate. Neither HRCT indices nor BAL findings differed significantly by AHR status.

Of the 42 patients whose long-term outcomes ( $\geqslant 12$ months) were available, seven were treated with systemic corticosteroids or an immunosuppressive agent for extrapulmonary lesions. Systemic corticosteroids were started for pulmonary sarcoidosis in another patient just after the comprehensive evaluation of airway. Of the remaining 34 patients in whom the natural course of pulmonary sarcoidosis was observed without systemic treatment, six (18\%) experienced pulmonary disease progression after the median (interquartile range) observational period of $41.5(12-78)$ months $(\geqslant 10 \%$ decline in forced vital capacity compared to baseline, $\geqslant 10 \%$ decline in forced expiratory volume in $1 \mathrm{~s}(\mathrm{FEV} 1)$ during the period). Demographic data and serum biomarkers at diagnostic baseline were similar between patients with progressive and stable disease. Among IOS and PFT indices, median (interquartile range) X5 and AX were significantly lower and higher in patients with progressive disease, respectively (X5: $-0.13(-0.72--0.11)$ versus $-0.09(-0.11--0.06$, $\mathrm{p}=0.01$; AX: $0.40(0.26-3.59)$ versus $0.16(0.11-0.27) ; \mathrm{p}=0.01)$, whereas PFT indices were comparable. Exhaled nitric oxide, HRCT indices and BAL findings did not differ between the groups.

We have shown that increased respiratory resistance is a better indicator for AHR in patients with early-stage pulmonary sarcoidosis than airflow limitation on PFTs. In a study of adult asthmatics, methacholine-induced dyspnoea and chest tightness did not correlate with changes in spirometry but did correlate with those in IOS [12]. Mildly asthmatic children have also displayed significantly higher IOS indices at baseline, relative to healthy controls, with no differences in FEV1 [13]. Another study of AHR in sarcoidosis revealed that decreased FEV1 predicted AHR although it did not include IOS measurements [14]. These results and our data both indicate the utility that IOS adds to even normal or mildly obstructive PFT profiles. Subjective respiratory symptoms such as cough, wheezing or dyspnoea on exertion were also typical accompaniments of AHR-positive sarcoidosis in our study. Given the low rate of airflow limitation in sarcoidosis [2], IOS may well serve as a screening tool for AHR in patients with early sarcoidosis, particularly if they have respiratory symptoms.

We have also demonstrated that decreased X5 and increased AX at the time of diagnosis are predictive for progression of pulmonary disease in patients with sarcoidosis. Although the determinants of X5 and AX values in IOS have not been fully defined, IOS may detect early airway disease and subtle parenchymal fibrosis without forced manoeuvres before these lesions have subsequently caused serious functional impairments on PFTs [4]. However, ventilation and lung volume may have affected these indices of IOS [10].

Some limitations of this study should be mentioned. First, the number of study subjects was small, and the follow-up period may not be enough to address the long-term outcome. Sarcoidosis was diagnosed only clinically without pathological confirmation in more than half of the subjects, which may have biased the study population. Another limitation was conducting this study at a single institute in Japan. Ethnical differences in clinical manifestations of sarcoidosis are well-known [15], and thus the prevalence of AHR and the long-term progression of pulmonary sarcoidosis may vary among differing ethnic groups. Finally, our patients did not receive uniform treatment, which may have skewed long-term outcomes of pulmonary lesions.

Despite these limitations, IOS has the potential to provide significant insight on AHR in early-stage pulmonary sarcoidosis. Reactance values may specifically predict the long-term progression of pulmonary involvement. Serial IOS measurements may also be useful to monitor the disease course.

Kiminobu Tanizawa ${ }^{1}$, Tomohiro $\mathrm{Handa}^{2}$, Sonoko Nagai ${ }^{3}$, Akio Niimi ${ }^{4}$, Tsuyoshi Oguma ${ }^{2}$, Takeshi Kubo $^{5}$, Yutaka Ito ${ }^{4}$, Kensaku Aihara ${ }^{6}$, Kohei Ikezoe ${ }^{2}$, Hisako Matsumoto ${ }^{2}$, Toyohiro Hirai ${ }^{2}$, Kazuo Chin ${ }^{1}$ and Michiaki Mishima ${ }^{1}$

${ }^{1}$ Dept of Respiratory Care and Sleep Control Medicine, Graduate School of Medicine, Kyoto University, Kyoto, Japan. ${ }^{2}$ Dept of Respiratory Medicine, Graduate School of Medicine, Kyoto University, Kyoto, Japan. ${ }^{3}$ Kyoto Central Clinic, Clinical Research Center, Kyoto, Japan. ${ }^{4}$ Dept of Medical Oncology and Immunology, Division of Respiratory Medicine, Graduate School of Medical Sciences, Nagoya City University, Nagoya, Japan. ${ }^{5}$ Dept of Diagnostic Imaging and Nuclear Medicine, Graduate School of Medicine, Graduate School of Medicine, Kyoto University, Kyoto, Japan. ${ }^{6}$ Dept of Respiratory Medicine, Saiseikai-Noe Hospital, Osaka, Japan.

Correspondence: Tomohiro Handa, Dept of Respiratory Medicine, Graduate School of Medicine, Kyoto University, 54 Shogoin Kawaharacho, Sakyo-ku, Kyoto 606-8507, Japan. E-mail: hanta@kuhp.kyoto-u.ac.jp

Received: Sept 282016 | Accepted: Dec 102016 
Support statement: This study was supported in part by a grant from Japan's Ministry of Health, Labor and Welfare to the Diffuse Lung Diseases Research Group, the Respiratory Failure Study Group, and the Research Program of Intractable Disease. Other support included the Japan Society for the Promotion of Science KAKENHI (grant numbers JP25860642, JP26461187 and JP16K09534) and a grant from the Japan Intractable Diseases Research Foundation and the Practical Research Project for Rare Intractable Diseases from Japan Agency for Medical Research and development, AMED (No. 15ek0109079h0001). The Dept of Respiratory Care and Sleep Control Medicine (Kyoto University, Kyoto, Japan) is funded by endowments from Philips Respironics, Teijin Pharma Ltd, Fukuda Denshi Inc. and Fukuda Lifetec Keiji. Funding information for this article has been deposited with the Open Funder Registry.

Conflict of interest: Disclosures can be found alongside this article at erjor.ersjournals.com

Acknowledgements: We appreciate M. Jinnai, K. Otsuka, T. Takeda, H. Nakaji, T. Tajiri, H. Inoue, T. Iwata, T. Nagasaki and Y. Kanemitsu (Dept of Respiratory Medicine, Graduate School of Medicine, Kyoto University, Kyoto, Japan) for the measurement of FeNO and T. Izumi (Kyoto Central Clinic, Clinical Research Center, Kyoto, Japan) for academic advice. We also thank T. Toki, N. Kimura and S. Tamura (Dept of Respiratory Care and Sleep Control Medicine, Graduate School of Medicine, Kyoto University, Kyoto, Japan) for help with manuscript preparation.

\section{References}

Polychronopoulos VS, Prakash UB. Airway involvement in sarcoidosis. Chest 2009; 136: 1371-1380.

2 Handa T, Nagai S, Fushimi Y, et al. Clinical and radiographic indices associated with airflow limitation in patients with sarcoidosis. Chest 2006; 130: 1851-1856.

3 Martusewicz-Boros MM, Boros WP, Wiatr E, et al. Bronchial hyperreactivity in sarcoidosis patients: correlation with airflow limitation indices. Sarcoidosis Vasc Diffuse Lung Dis 2012; 29: 99-106.

4 Bickel S, Popler J, Lesnick B, et al. Impulse oscillometry: interpretation and practical applications. Chest 2014; 146 : $841-847$.

5 Handa T, Nagai S, Ito I, et al. Cytotoxic T-lymphocyte antigen-4 (CTLA-4) exon 1 polymorphism affects lymphocyte profiles in bronchoalveolar lavage of patients with sarcoidosis. Sarcoidosis Vasc Diffuse Lung Dis 2003; 20: $190-196$

6 American Thoracic Society, European Respiratory Society. ATS/ERS recommendations for standardized procedures for the online and offline measurement of exhaled lower respiratory nitric oxide and nasal nitric oxide, 2005. Am J Respir Crit Care Med 2005; 171: 912-930.

7 Hagmolen of ten Have W, van den Berg NJ, van der Palen J, et al. Validation of a single concentration methacholine inhalation provocation test (SCIPT) in children. J Asthma 2005; 42: 419-423.

8 Merget R, Jörres RA, Heinze E, et al. Development of a 1-concentration-4-step dosimeter protocol for methacholine testing. Respir Med 2009; 103: 607-613.

9 Judson MA, Baughman RP, Teirstein AS, et al. Defining organ involvement in sarcoidosis: the ACCESS proposed instrument. ACCESS Research Group. A case control etiologic study of sarcoidosis. Sarcoidosis Vasc Diffuse Lung Dis 1999; 16: 75-86.

10 Tanimura K, Hirai T, Sato S, et al. Comparison of two devices for respiratory impedance measurement using a forced oscillation technique: basic study using phantom models. J Physiol Sci 2014; 64: 377-382.

11 Ohara T, Hirai T, Sato S, et al. Comparison of airway dimensions in different anatomic locations on chest CT in patients with COPD. Respirology 2006; 11: 579-585.

12 Mansur AH, Manney S, Ayres JG. Methacholine-induced asthma symptoms correlate with impulse oscillometry but not spirometry. Respir Med 2008; 102: 42-49.

13 Kim HY, Shin YH, Jung DW, et al. Resistance and reactance in oscillation lung function reflect basal lung function and bronchial hyperresponsiveness respectively. Respirology 2009; 14: 1035-1041.

14 Young LM, Good N, Milne D, et al. The prevalence and predictors of airway hyperresponsiveness in sarcoidosis. Respirology 2012; 17: 653-659.

15 Baughman RP, Nagai S, Balter M, et al. Defining the clinical outcome status (COS) in sarcoidosis: results of WASOG Task Force. Sarcoidosis Vasc Diffuse Lung Dis 2011; 28: 56-64. 